\title{
Title:
}

\section{Small-bowel transit time in capsule endoscopy: a determinant factor for the diagnosis of small-bowel bleeding}

\section{Authors:}

Cátia Arieira, Francisca Dias de Castro, Pedro Boal Carvalho, Bruno Rosa, Maria João Moreira, José Cotter

DOI: $10.17235 /$ reed.2021.7487/2020

Link: PubMed (Epub ahead of print)

Please cite this article as:

Arieira Cátia, Dias de Castro Francisca, Boal Carvalho Pedro, Rosa Bruno, Moreira Maria João, Cotter José. Smallbowel transit time in capsule endoscopy: a determinant factor for the diagnosis of small-bowel bleeding. Rev Esp Enferm Dig 2021. doi: 10.17235/reed.2021.7487/2020.

This is a PDF file of an unedited manuscript that has been accepted for publication. As a service to our customers we are providing this early version of the manuscript. The manuscript will undergo copyediting, typesetting, and review of the resulting proof before it is published in its final form. Please note that during the production process errors may be discovered which could affect the content, and all legal disclaimers that apply to the journal pertain. 
OR 7487

Small-bowel transit time in capsule endoscopy: a determinant factor for the diagnosis of small-bowel bleeding

Cátia Arieira ${ }^{1,2,3}$, Francisca Dias de Castro ${ }^{1,2,3}$, Pedro Boal Carvalho ${ }^{1,2,3}$, Bruno Rosa ${ }^{1,2,3}$, Maria João Moreira ${ }^{1,2,3}$, José Cotter ${ }^{1,2,3}$

${ }^{1}$ Gastroenterology Department. Hospital da Senhora da Oliveira. Guimarães, Portugal. ${ }^{2}$ Instituto de Investigação em Ciências da Vida e Saúde (ICVS). Escola de Medicina. Universidade do Minho. Braga, Portugal. ${ }^{3}$ Laboratório Associado ICVS/3B's. Braga/Guimarães, Portugal

Received: 02/09/2020

Accepted: 10/01/2021

Correspondence: Cátia Arieira. Gastroenterology Department. Hospital da Senhora da Oliveira. Rua dos Cutileiros, Creixomil. 4835-044 Guimarães, Portugal e-mail: catia_arieira@hotmail.com

Author contributions: Arieira C. and Dias de Castro F. designed the study, interpreted the data, and drafted the manuscript. Arieira C., Boal Carvalho P., Rosa B., and Moreira MJ reviewed the small-bowel capsule endoscopies. Arieira C. participated in the acquisition of data and performed the statistical analysis. Dias de Castro F., Boal Carvalho P., Rosa B., and Moreira MJ. critically revised the manuscript. Cotter J. critically revised and approved the final version of the manuscript.

Disclosures: Rosa B. has a consulting services agreement with Medtronic ${ }^{\circledR}$. Other authors have no conflicts of interest to declare.

\section{ABSTRACT}


Background: small-bowel capsule endoscopy (SBCE) is the gold standard for the study of small-bowel bleeding (SBB). Recent studies suggest that longer small-bowel transit times (SBTT) may be associated with a higher diagnostic yield of SBCE.

Aim: the aim of the study was to investigate if longer SBTT is a predictive factor of positive findings on SBCE in a population that underwent SBCE for suspected SBB.

Methods: a retrospective single-center study including consecutive SBCEs between May 2012 and May 2019, due to suspected SBB. A positive SBCE was considered in the presence of lesions with high bleeding potential such as ulcers, angioectasias, and tumors (P2 lesions, according to the Saurin classification).

Results: we included 372 patients, 65.9 \% female, with a median age of 67 (IQR: 19-97) years. We observed that patients with P2 lesions ( $n=131 ; 35.2 \%$ ) in SBCE exhibited a longer SBTT $(p=0.01)$, were older $(p<0.001)$, were more frequently male $(p=0.019)$, and suffered more frequently from arterial blood hypertension $(p=0.011)$, diabetes ( $p$ $=0.042)$, chronic kidney disease $(p=0.003)$, and heart failure $(p=0.001)$. In the logistic analysis, significant predictive factors for the presence of $P 2$ lesions included age (OR: 1.027; 95 \% Cl: 1.009-1.045; $\mathrm{p}=0.004$ ), SBTT (OR: 1.002; 95 \% Cl: 1.001-1.005; $\mathrm{p}=$ 0.029 ), and male gender (OR: 1.588; $95 \% \mathrm{Cl}: 1.001-2.534 ; \mathrm{p}=0.049$ ).

Conclusions: patients with longer SBTT had higher rates of lesions with high bleeding potential (P2). SBTT along with previously well-defined factors such as age and male gender were the only independent predictive factors for the presence of P2 lesions. These findings may suggest that a slower passage of the capsule through the small bowel may allow a better diagnostic yield for significant lesions.

Keywords: Capsule endoscopy. Small intestine.

\section{BACKGROUND}

Small-bowel bleeding (SBB) accounts for $5 \%$ of all intestinal bleeding, and the source is defined as distal to the ampulla of Vater and proximal to the ileocecal valve (1). Since 2001, small-bowel capsule endoscopy (SBCE) constitutes the gold-standard 
investigation for SBB $(1,2)$. It is a noninvasive tool that provides direct visualization of the entire small-bowel mucosa, with an excellent diagnostic yield and high negative predictive value ( $83 \%$ to $100 \%$ ) (1). This tool can be used as a screening method in the identification of bleeding lesions, with the ability to guide future therapeutic endoscopic approaches such as device-assisted enteroscopy $(1,2)$. Several small-bowel lesions may be responsible for SBB, most commonly vascular lesions, predominantly angioectasias, while other sources include inflammation from Crohn's disease, nonsteroidal (NSAID) enteropathy, tumors, Meckel's diverticula, and other rare etiologies (3).

The diagnostic yield of SBCE may be influenced by multiple factors. There is a higher likelihood of positive findings in patients with older age ( $>65$ years), overt bleeding, use of anticoagulant, antiplatelet, or non-steroidal anti-inflammatory drugs (NSAIDs), higher transfusion requirements, and chronic comorbidities such as dyslipidemia and hypertension (4-7). Recently, small-bowel transit time (SBTT) was highlighted as a factor associated with the diagnostic yield of significant lesions in SBB (8-10). Incomplete SBCE evaluation may impair diagnostic yield, and factors such as hospitalization, previous surgery or radiation, diabetes mellitus, and very old age have been identified as potential risk factors. The combined strategy of checking the position of the capsule with a real-time viewer and selectively administering prokinetics has been adopted to overcome this limitation (11).

The aim of the study was to investigate if a longer SBTT is a predictive factor of positive significant findings on SBCE in a population that underwent SBCE for suspected SBB.

\section{METHODS}

This study was a single-center retrospective study. All patients referred to our center to undergo SBCE for suspected SBB between May 2012 and May 2019 were reviewed. SBB was characterized as overt in the presence of melena or hematochezia, or as occult if manifested as iron deficiency anemia or a positive fecal occult blood test. All patients underwent nondiagnostic esophagogastroduodenoscopy and total colonoscopy with adequate bowel preparation prior to SBCE. Furthermore, females were evaluated to exclude abnormal gynecological bleeding. The patients' clinical 
information was collected from medical records, including gender, age, presentation (overt vs occult) of SBB, and comorbidities, namely hypertension, dyslipidemia, diabetes, chronic kidney disease, heart failure, and medical therapy.

\section{SBCE procedure}

SBCE was performed using a PillCam ${ }^{\circledR}$ SB2 or SB3 (Given ${ }^{\circledR}$ Imaging Ltd. Yoqneam, Israel).

Patients that underwent SBCE between May 2012 and February 2018 had a clear liquid diet for $24 \mathrm{~h}$ and a 12-h fast prior to SBCE, and were advised not to eat for $4 \mathrm{~h}$ after swallowing the capsule (Protocol A). From February 2018 to May 2019, patients followed a 24-h clear-liquid diet and a 12-h fast prior to SBCE, and 1 L of Moviprep ${ }^{\circledR}$ was administered after real-time confirmation of capsule arrival at the small bowel (Protocol B). For all procedures, patients were given $100 \mathrm{mg}$ of simethicone $30 \mathrm{~min}$ before capsule ingestion, and $1 \mathrm{~h}$ after ingestion they returned to our unit for real-time visualization using a real-time viewer. At this point, if the capsule remained in the stomach, the patient was given $10 \mathrm{mg}$ of oral domperidone. Thirty minutes after prokinetic administration, if the capsule remained in the stomach, it was placed into the duodenum by upper gastrointestinal endoscopy.

Every patient who underwent SBCE signed an informed consent, and all the data collected were coded to ensure anonymity. The ethical committee of Gastroenterology Department, Hospital da Senhora da Oliveira - Guimarães approved the study.

\section{Exclusion criteria}

Patients with incomplete SBCE or inadequate small-bowel preparation were excluded. Inadequate small-bowel preparation was defined when the mucosa could be observed in $<50 \%$ of recording time, with the presence of significant amounts of fluid, bubbles, or debris that compromised the interpretation of the examination (12).

\section{Findings}

Findings were classified using the Capsule Endoscopy Standard Terminology. A positive SBCE was considered in the presence of lesions with a high bleeding potential, or 
luminal blood-P2, according to the Saurin classification (13).

\section{Small-bowel transit time}

SBTT was calculated in minutes and defined as the time elapsed from the first frame of duodenal bulb to the first frame of the cecum.

\section{Statistical analysis}

The statistical analysis was performed with the SPSS ${ }^{\circledR}$, version 22.0 (IBM, Armonk, New York, USA). Categorical variables are presented as frequencies and percentages, and continuous variables as means, medians, standard deviations, and range. All reported $\mathrm{p}$-values are two tailed, with a p-value less than 0.05 indicating statistical significance. The distribution of categorical variables between groups was evaluated by $\chi^{2}$ analysis, and continuous variables with the Mann-Whitney U-test. A multivariate analysis was performed using binomial regression to identify potential independent predictive factors of a positive SBCE. Spearman's rank correlation coefficient was used to calculate the correlation between factors apt to influence SBTT. A receiver operating characteristic (ROC) analysis was performed to evaluate the performance of SBTT to predict positive SBCE, and Youden's index was used to find the optimal cut-off for SBTT.

\section{RESULTS}

Four hundred and seven patients underwent SBCE during this period; 35 were excluded due to incomplete SBCE $(n=24)$ or inadequate small-bowel preparation $(n=$ 11). Thus, 372 patients were finally included, of whom $65.9 \%$ were female with a median age of 67 (IQR: 19-97) years. Out of this population, $16.4 \%(n=61)$ underwent SBCE during hospitalization. Regarding the type of small-bowel preparation, $80.1 \%$ (n $=298)$ underwent Protocol $A$ and $19.9 \%(n=74)$ underwent Protocol B. From this population, $79.3 \%$ underwent SBCE due to occult bleeding, and $20.7 \%$ due to overt bleeding; P2 lesions were present in 131 (35.2\%) patients. These patients had a median SBTT of 267 (55-670) minutes. 
Regarding patients with P2 lesions on SBCE, $85.5 \%(n=112)$ presented angioectasias, $9.9 \%(n=13)$ ulcerations, $3.8 \%(n=5)$ tumors, and $0.76 \%(n=1)$ varices. Patients with P2 lesions on SBCE had longer SBTTs, were older, were more frequently male, and suffered more frequently from hypertension, diabetes, chronic kidney disease, or heart failure. Concerning the presentation of SBB (occult vs overt), no differences were observed between the two groups. Furthermore, no differences were observed between patients under anticoagulants $(p=0.598)$ and on antiplatelet agents $(p=$ 0.553).

Regarding the factors that may influence SBTT, there was a significant correlation between SBTT and age ( $r$ : 0.136; $p=0.008$ ) (Fig. 1). SBCEs with SBTT $\geq 321 \mathrm{~min}$ presented higher rates of P2 lesions ( $47.0 \%$ vs $29.8 \%$; $p=0.001)$, with a sensitivity of $41.98 \%$ and specificity of $74.27 \%$. Figure 2 shows the ROC curve of SBTT to predict the presence of P2 lesions with an AUROC of 0.581 (95\% Cl: 0.52-0.642). Concerning small-bowel preparation, the administration of purgatives (Protocol B) (median: 283 [86-619] versus 263 [55-670] $\mathrm{min} ; p=0.255)$, gender $(p=0.979)$, the presence of diabetes $(p=0.963)$, and inpatient status (median: 279 [86-670] versus 261 [55-653] years; $p<0.616)$ did not influence SBTT. According to the logistic regression, the significant predictive factors for the presence of $P 2$ lesions were age $(p=0.004)$, SBTT $(p=0.029)$, and male gender $(p=0.049)$. Univariate and logistic regression parameters are presented in table 1.

\section{DISCUSSION}

Over the years, several studies have assessed risk factors associated with a positive SBCE on SBB. Older age, overt bleeding, anticoagulant use, antiplatelet use, NSAID use, lower hemoglobin, higher transfusion requirements, and patients with chronic comorbidities such as chronic kidney disease, dyslipidemia, and hypertension are associated with a higher diagnostic yield of SBB (4-7).

Recent studies have hypothesized that longer SBTTs could be a factor associated with higher rates of lesion detection in SBB (8-10). In our cohort, the univariate analysis showed, in accordance to previous studies, that older patients, male gender, and patients with chronic comorbidities such as hypertension, diabetes, CKD, and HF 
presented more frequently with P2 lesions on SBCE (4-7). Moreover, in our study, patients with P2 lesions by SBCE had longer SBTTs, suggesting that a slower passage of the capsule through the small bowel allows a better inspection of the small-bowel mucosa and is associated with a higher diagnostic yield for significant lesions (8).

Furthermore, except for male gender and older age, according to the logistic regression, SBTT constitutes an independent predictive factor for the presence of P2 lesions. In addition, older patients had a longer SBTT, likely due to the reduced motility that occurs with aging, which partially explains the higher diagnostic yield of P2 lesions in this population. This is in line with previous series $(8,14)$.

In accordance with our results, Girelli C. et al. found that longer SBTT and older age were associated with higher rates of P2 lesions (8). Blanco-Velasco and coworkers suggested that a SBTT $\geq 4$ hours was associated with an improved diagnostic yield with an odds ratio of 3.13 (10). Egea-Valenzuela J. et al. reported a positive correlation between prolonged SBTT and higher diagnostic yield of SBCE. However, this study included all types of indications for SBCE and not only SBB (9). Conversely, VelayosJiménez B. et al. found no differences in SBTT with regard to age, gender, or body mass index despite their study being limited by sample size $(n=89)$ and a younger mean age of their patients $(59.20 \pm 17.27)(15)$.

Regarding the presence of comorbidities, a study of 29 patients found that diabetics had a prolonged gastric transit time and shorter SBTT. However, we did not find such an association in our study, and SBTT did not correlate with diabetes status (16). This finding may be explained by our preparation protocol, which includes the administration of prokinetics to overcome the long standing of the capsule in the stomach. Some authors raised the question about the use of prokinetics and purgatives during SBCE since, in theory, some of these products may reduce SBTT and consequently impact on the procedure's diagnostic yield (8).

Adler et al. presented conflicting results regarding the use of small-bowel preparation, and reported a better visibility of the small bowel when using a post-ingestion purgebased cleansing protocol (Picolax ${ }^{\circledR}$ ), although with a shorter SBTT (17). On the contrary, in our study and in accordance to a previous study in our center, the administration of bowel preparation (PEG $1 \mathrm{~L}$ ) did not shorten the SBTT, and had no 
influence on diagnostic yield (18).

In addition, several authors reported that inpatient status is inversely correlated with completion rate of SBCE, mainly because hospitalized patients represent a population with reduced mobility and worse overall health (19-21). Notably, in our study we did not find a significantly longer SBTT in hospitalized patients, and it could be explained by a population selection bias, since we excluded patients with incomplete SBCE.

Regarding study limitations, this is a retrospective study and we were unable to identify the proportion of patients who needed prokinetic administration during the procedure. Thus, we cannot compare SBTT between these groups. Even so, a previous study by our group found that the selective administration of prokinetics, namely domperidone, guided by the position of the capsule in a real-time viewer, is a valid approach towards reducing incomplete examinations, with no effect on SBTT or diagnostic yield (11).

In conclusion, we found that a longer SBTT in SBB is independently associated with a higher diagnostic yield, namely the identification of lesions with a higher bleeding potential. Age is the main determinant influencing SBTT, and it does not seem to be influenced by prokinetics or purgative solutions. Thus, these can be safely used to increase the proportion of complete examinations and the quality of small-bowel preparation, while not compromising SBTT and consequently the diagnostic yield of SBCE in patients presenting with SBB. 


\section{REFERENCES}

1. Gerson LB, Fidler JL, Cave DR, et al. ACG Clinical Guideline: Diagnosis and Management of Small Bowel Bleeding. Am J Gastroenterol 2015;110:1265-87. DOI: 10.1038/ajg.2015.246

2. Rondonotti E, Spada C, Adler S, et al. Small-bowel capsule endoscopy and device-assisted enteroscopy for diagnosis and treatment of small-bowel disorders: European Society of Gastrointestinal Endoscopy (ESGE) Technical Review. Endoscopy 2018;50:423-46. DOI: 10.1055/a-0576-0566

3. Gunjan D, Sharma V, Rana SS, et al. Small bowel bleeding: a comprehensive review. Gastroenterol Rep (Oxf) 2014;2:262-75. DOI: 10.1093/gastro/gou025

4. Boal Carvalho P, Rosa B, Moreira MJ, et al. New evidence on the impact of antithrombotics in patients submitted to small bowel capsule endoscopy for the evaluation of obscure gastrointestinal bleeding. Gastroenterol Res Pract 2014;2014:709217. DOI: 10.1155/2014/709217

5. Cúrdia Gonçalves T MJ, Boal Carvalho P, Moreira MJ, et al. Is It Possible to Predict the Presence of Intestinal Angioectasias? Diagn Ther Endosc 2014;461602. DOI: 10.1155/2014/461602

6. Katsinelos $P$, Kountouras $J$, Chatzimavroudis $G$, et al. Factors predicting a positive capsule endoscopy in past overt obscure gastrointestinal bleeding: a multicenter retrospective study. Hippokratia 2016;20:127-32.

7. Ribeiro I, Pinho R, Rodrigues A, et al. Obscure gastrointestinal bleeding: Which factors are associated with positive capsule endoscopy findings? Rev Esp Enferm Dig 2015;107:334-9.

8. Girelli CM, Soncini M, Rondonotti E. Implications of small-bowel transit time in the detection rate of capsule endoscopy: A multivariable multicenter study of patients with obscure gastrointestinal bleeding. World J Gastroenterol 2017;23:697-702. DOI: 10.3748/wjg.v23.i4.697

9. Egea Valenzuela J, Sanchez Martinez A, Garcia Marin AV, et al. Influence of demographic and clinical features of the patient on transit times and impact the on the diagnostic yield of capsule endoscopy. Rev Esp Enferm Dig 
2019;111:530-6. DOI: 10.17235/reed.2019.5971/2018

10. Blanco Velasco G, Álvarez Licona NE. Small bowel transit time of capsule endoscopy as a factor for the detection of lesions in potential small bowel bleeding. Rev Esp Enferm Dig 2019;111(9):696-8. DOI: $10.17235 /$ reed.2019.5943/2018

11. Cotter J, de Castro FD, Magalhaes J, et al. Finding the solution for incomplete small bowel capsule endoscopy. World J Gastrointest Endosc 2013;5:595-9. DOI: 10.4253/wjge.v5.i12.595

12. Rosa BJ, Barbosa M, Magalhaes J, et al. Oral purgative and simethicone before small bowel capsule endoscopy. World J Gastrointest Endosc 2013;5:67-73. DOI: $10.4253 /$ wjge.v5.i2.67

13. Saurin JC, Delvaux M, Gaudin JL, et al. Diagnostic value of endoscopic capsule in patients with obscure digestive bleeding: blinded comparison with video pushenteroscopy. Endoscopy 2003;35:576-84. DOI: 10.1055/s-2003-40244

14. Fireman Z, Kopelman Y, Friedman S, et al. Age and indication for referral to capsule endoscopy significantly affect small bowel transit times: the given database. Dig Dis Sci 2007;52:2884-7. DOI: 10.1007/s10620-007-9789-1

15. Velayos Jiménez B, Aller de la Fuente R, de la Calle Valverde F, et al. Study of gastronitestinal transit times with capsule endoscopy. Gastroenterol Hepatol 2005;28(6):315-20. DOI: 10.1157/13076347

16. Triantafyllou K, Kalantzis C, Papadopoulos AA, et al. Video-capsule endoscopy gastric and small bowel transit time and completeness of the examination in patients with diabetes mellitus. Dig Liver Dis 2007;39:575-80. DOI: 10.1016/j.dld.2007.01.024

17. Adler SN, Farkash S, Sompolinsky Y, et al. A novel purgative protocol for capsule endoscopy of the small bowel produces better quality of visibility than 2 I of PEG: Timing is of the essence. United European Gastroenterol J 2017;5:485-90. DOI: $10.1177 / 2050640616665291$

18. Xavier S, Rosa B, Monteiro S, et al. Bowel preparation for small bowel capsule endoscopy - The later, the better! Dig Liver Dis 2019;51:1388-91. 
19. Yazici C, Losurdo J, Brown MD, et al. Inpatient capsule endoscopy leads to frequent incomplete small bowel examinations. World J Gastroenterol 2012;18:5051-7. DOI: 10.3748/wjg.v18.i36.5051

20. Westerhof J, Weersma RK, Koornstra JJ. Risk factors for incomplete small-bowel capsule endoscopy. Gastrointest Endosc 2009;69:74-80. DOI: 10.1016/j.gie.2008.04.034

21. Robinson CA, Jackson C, Condon D, et al. Impact of inpatient status and gender on small-bowel capsule endoscopy findings. Gastrointest Endosc 2011;74:1061-6. DOI: 10.1016/j.gie.2011.07.019 
Table 1. Factors associated with a positive SBCE (P2 lesions)

\begin{tabular}{|l|l|l|l|l|l|l|}
\hline & \multicolumn{4}{l}{ Univariate analysis } & \multicolumn{3}{l|}{ Binomial regression } \\
\hline & P2 lesions & no P2 lesions & $\mathbf{p}$ & OR & $\mathbf{9 5} \% \mathrm{Cl}$ & $\mathbf{p}$ \\
\hline SBTT (min) & 295 & 256 & $0.01^{*}$ & 1.002 & $1.001-1.005$ & $0.029^{*}$ \\
\hline Age & 73.0 & 60.0 & $<0.001^{*}$ & 1.027 & $1.009-1.045$ & $0.004^{*}$ \\
\hline Male gender & $42.0 \%$ & $29.9 \%$ & $0.019^{*}$ & 1.588 & $1.001-2.534$ & $0.049^{*}$ \\
\hline Hypertension & $69.5 \%$ & $56.0 \%$ & $0.011^{*}$ & & & 0.399 \\
\hline Diabetes & $38.2 \%$ & $28.6 \%$ & $0.042^{*}$ & & & 0.712 \\
\hline CKD & $34.4 \%$ & $20.3 \%$ & $0.003^{*}$ & & & 0.245 \\
\hline HF & $38.2 \%$ & $21.6 \%$ & $0.001^{*}$ & & & 0.369 \\
\hline Overt vs occult & $44.2 \%$ & $32.9 \%$ & 0.065 & --- & ---- & ---- \\
\hline Anticoagulants & $21.4 \%$ & $19.1 \%$ & 0.598 & --- & --- & ---- \\
\hline Antiplatelets & $35.1 \%$ & $32.1 \%$ & 0.553 & --- & ---- & ---- \\
\hline & & & & & & \\
\hline
\end{tabular}




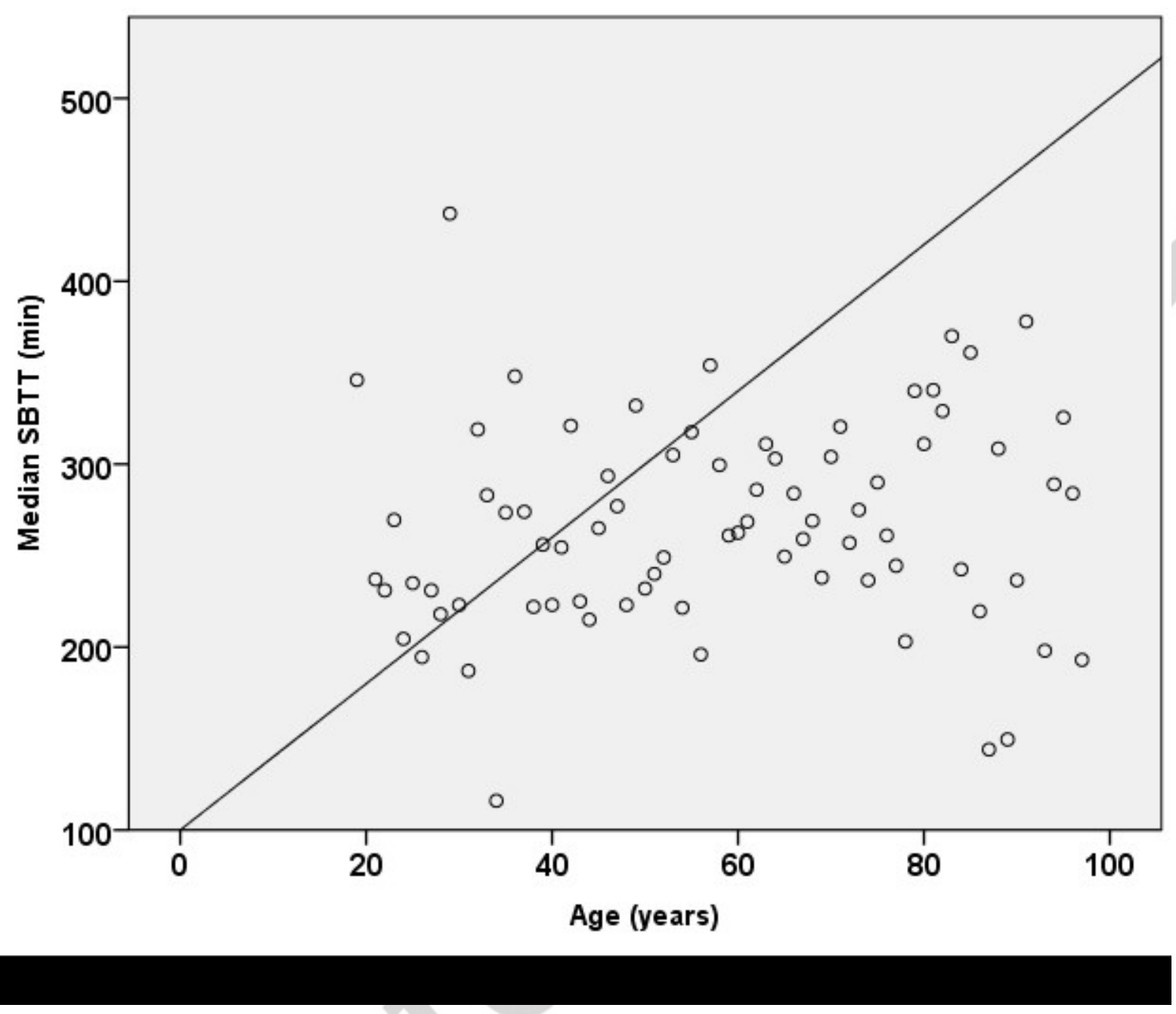

Fig. 1. Spearman's correlation between SBTT ( $\mathrm{min}$ ) and age (years). 


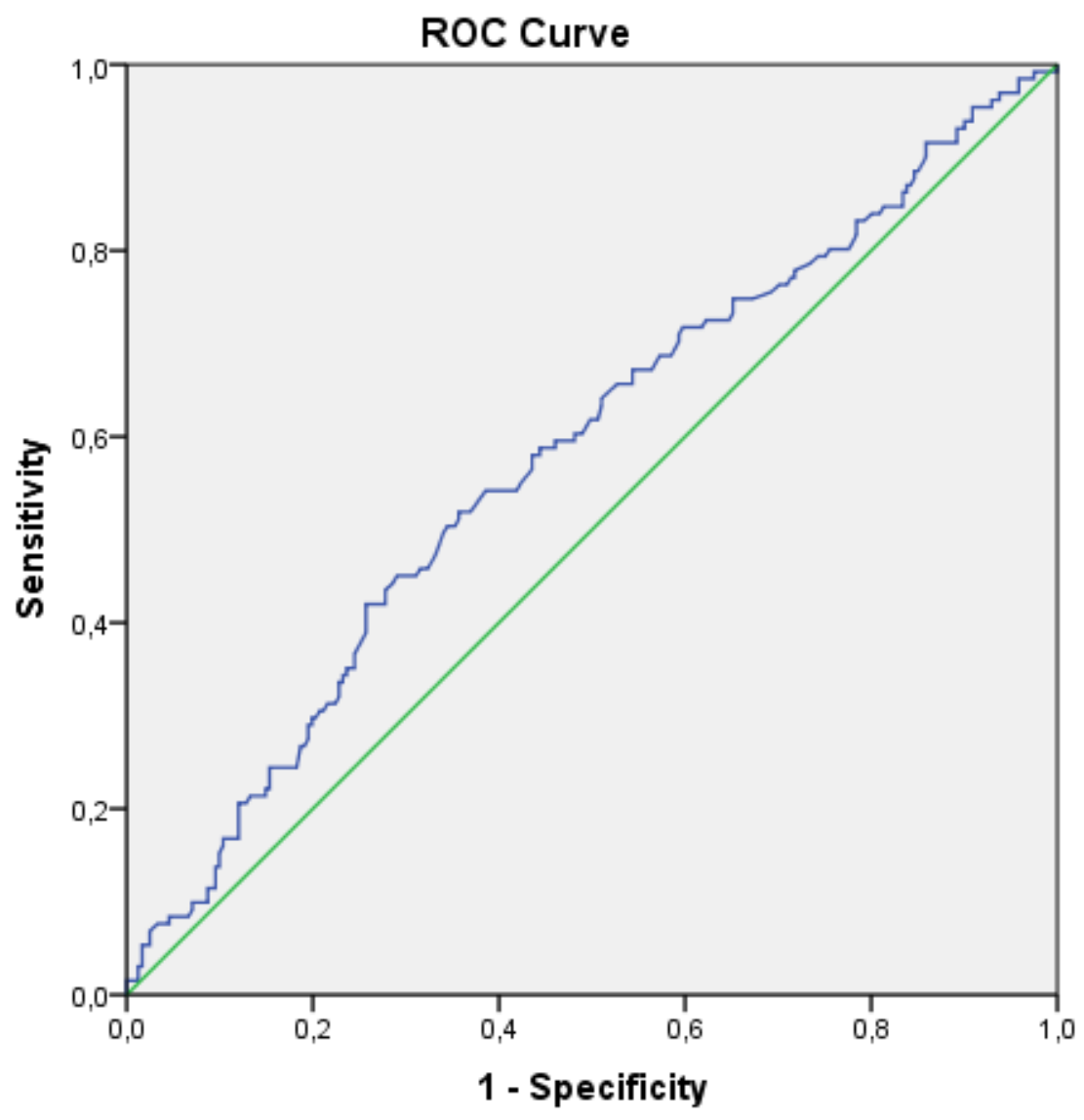

Diagonal segments are produced by ties.

Fig. 2. ROC curve of SBTT to predict the presence of P2 lesions (AUROC $=0.581$; cut-off = 321 min; sensitivity: $41.98 \%$; specificity: $74.27 \%$ ). 